\title{
HUREWICZ FIBRATIONS NEED NOT BE LOCALLY TRIVIAL
}

\author{
L. S. $\mathrm{HUSCH}^{1}$
}

Abstract. A Hurewicz fibration, $p: M \rightarrow S^{1}$, from a closed manifold onto the 1 -sphere is constructed which is not locally trivial.

A map $p: E \rightarrow B$ is a Hurewicz fibration if $p$ possesses the covering homotopy property for all spaces; i.e., given a homotopy $H: X \times[0,1] \rightarrow B$ and a map $h: X \rightarrow E$ such that $p h(x)=H(x, 0), x \in X$, there exists a homotopy $G: X \times[0,1] \rightarrow E$ such that $G(x, 0)=h(x), x \in X$, and $p G(x, t)$ $=H(x, t),(x, t) \in X \times[0,1] . p: E \rightarrow B$ is locally trivial if, for each $x \in B$, there exists a neighborhood $U$ of $x$ in $B$ and a homeomorphism $h: U \times p^{-1}(x)$ $\rightarrow p^{-1}(U)$ such that $p h(y, z)=y$ for all $(y, z) \in U \times p^{-1}(x)$. F. Raymond [4] conjectured that a Hurewicz fibration, $p: E \rightarrow B$, of a closed manifold $E$ onto a weakly locally contractible paracompact space $B$ is locally trivial. He was able to prove the conjecture when there exists some compact fiber of dimension not greater than two. In this note, we show that the conjecture is false when the dimension of the fiber is greater than two.

Let $S^{n}$ be the $n$-sphere, $n \geqslant 3$, and let $\alpha \subseteq S^{n}$ be a noncellular arc. Let $M$ be the decomposition space obtained from $S^{n} \times S^{1}$ by shrinking $\alpha \times\left\{x_{0}\right\}$ to a point for some fixed $x_{0} \in S^{1}$. Let $f: S^{n} \times S^{1} \rightarrow M$ be the natural projection; then there exists a unique map $p: M \rightarrow S^{1}$ such that $p f(x, y)=y$ for all $(x, y) \in S^{n} \times S^{1}$. Note that $\alpha \times\left\{x_{0}\right\}$ is cellular in $S^{n} \times S^{1}$ and, hence, $M$ is homeomorphic to $S^{n} \times S^{1}$. Clearly, $p$ is not locally trivial.

We will now show that $p$ is a Hurewicz fibration. Let $Q$ denote the Hilbert cube; we use the following result of T. Chapman and S. Ferry [1] which is a parameterized version of a result of R. D. Edwards [2].

TheOREM. Let $E$ and $B$ be $A N R$ 's, $p: E \rightarrow B$ a map and let $M$ be a $Q$ manifold. If $k: M \times B \rightarrow E$ is a fiber-preserving $C E$ map, then $k \times \mathrm{id}: M \times B$ $\times Q \rightarrow E \times Q$ is a fiber-preserving near homeomorphism.

Define $k: S^{n} \times Q \times S^{1} \rightarrow M$ by $k(x, y, z)=f(x, z)$. Since $p k(x, y, z)=z, k$ is fiber-preserving; note also that $k$ is $C E$; i.e. if $x \in M$, then $k^{-1}(x)$ has property $U V^{\infty}$ [3]. By the theorem, $k \times$ id: $S^{n} \times Q \times S^{1} \times Q \rightarrow M \times Q$ is a fiber-preserving near homeomorphism; in particular, there exists a homeomor-

Received by the editors April 5, 1976.

AMS (MOS) subject classifications (1970). Primary 55F05; Secondary 55F10, 54B15, 57A20.

${ }^{1}$ Research was supported in part by N.S.F. Grant MPS 72-04690. 
phism $\phi: S^{n} \times Q \times S^{1} \times Q \rightarrow M \times Q$ such that $p \mu \phi(x, y, z, w)=z$, where $\mu: M \times Q \rightarrow M$ is the projection. Let $\gamma: S^{n} \times Q \times S^{1} \times Q \rightarrow S^{1}$ also be projection.

Let $H: X \times[0,1] \rightarrow S^{1}$ and $h: X \rightarrow M$ be maps such that $p h(x)=H(x, 0)$. Define $h^{\prime}: X \rightarrow S^{n} \times Q \times S^{1} \times Q$ by $h^{\prime}(x)=\phi^{-1}(h(x), 0)$. Since $\gamma h^{\prime}(x)$ $=p h(x)=H(x, 0)$, there exists $G^{\prime}: X \times[0,1] \rightarrow S^{n} \times Q \times S^{1} \times Q$ such that $G^{\prime}(x, 0)=h^{\prime}(x)$ and $\gamma G^{\prime}(x, t)=H(x, t) . G(x, t)=\mu \phi G^{\prime}(x, t)$ is the desired homotopy.

\section{REFERENCES}

1. T. Chapman and S. Ferry, Hurewicz fiberings of ANR's (to appear).

2. R. D. Edwards (to appear).

3. R. C. Lacher, Cell-like mappings. I, Pacific J. Math. 30 (1969), 717-731. MR 40 \#4941.

4. F. Raymond, Local triviality for Hurewicz fiberings of manifolds, Topology 3 (1965), 43-57. MR 28 \#2554; 29, p. 1437.

Department of Mathematics, University of Tennessee, Knoxville, Tennessee 37916

Current address: Department of Mathematics, University of Zagreb, P.P.187, Zagreb, Yugoslavia 